\section{D contrast-enhanced lung perfusion MRI is an effective screening tool for chronic thromboembolic pulmonary hypertension: results from the ASPIRE Registry}

Background Chronic thromboembolic pulmonary hypertension (CTEPH) is a complication of pulmonary embolism potentially curable by surgery. Perfusion scintigraphy is currently advocated as the imaging modality of choice to exclude CTEPH due to its high sensitivity. We have evaluated the diagnostic utility of lung perfusion MRI.

Methods Consecutive patients attending a pulmonary hypertension referral centre undergoing lung perfusion MRI, perfusion scintigraphy, CT pulmonary angiography (CTPA) and right heart catheterisation within 14 days were identified.

Results of 132 patients, 78 were diagnosed as having CTEPH. Lung perfusion MRI correctly identified 76 patients as having CTEPH with an overall sensitivity of $97 \%$, specificity $92 \%$, positive predictive value $95 \%$ and negative predictive value $96 \%$ compared with perfusion scintigraphy (sensitivity $96 \%$, specificity 90\%) and CTPA (sensitivity 94\%, specificity $98 \%$ ). No cases of surgically accessible CTEPH were missed with either modality.
Conclusions Lung perfusion MRI has high sensitivity equivalent to perfusion scintigraphy in diagnosing CTEPH but does not require ionising radiation, making it an attractive initial imaging modality to assess patients with suspected CTEPH.

Chronic thromboembolic pulmonary hypertension (CTEPH) occurs in up to $3.8 \%$ of patients following pulmonary thromboembolism (PTE) and is a major cause of severe pulmonary hypertension (PH). Patients usually present with breathlessness following PTE or with unexplained PH. The diagnosis may be missed on CT pulmonary angiography (CTPA) by radiologists not experienced in pulmonary vascular disease, so perfusion scintigraphy has been recommended as a screening test, given its high sensitivity. In recent years, MRI has evolved into a holistic imaging modality in $\mathrm{PH}$, allowing morphological assessment of the pulmonary vasculature and structural and functional assessment of the heart. ${ }^{1}$ In addition, 3D contrast-enhanced lung perfusion MRI provides insight into regional pulmonary perfusion by tracking the dynamic passage of a contrast bolus. Previous studies have demonstrated the feasibility of this technique in acute PTE. ${ }^{2}$ However, there is limited data on the performance of 3D contrast-enhanced lung perfusion MRI in diagnosing CTEPH in a high-risk population. $^{34}$
From a large PH referral centre, consecutive patients with suspected CTEPH or unexplained $\mathrm{PH}$ who had lung perfusion MRI, perfusion scintigraphy, CTPA and right heart catheterisation within 14 days were identified from the ASPIRE registry $^{5}$ to compare the diagnostic utility of these imaging modalities. 3D MR lung perfusion images were acquired using a time resolved 3D spoiled gradient echo sequence with view sharing and were analysed by two radiologists blinded to the other imaging studies and clinical information. The final diagnosis of CTEPH was based on clinical assessment, right heart catheter and imaging, including MR angiographic studies (but not the MR perfusion images analysed in this study), CTPA and perfusion scintigraphy. The diagnosis of surgically accessible disease was confirmed at the national pulmonary endarterectomy centre at Papworth.

Of the 132 patients fulfilling the inclusion criteria, 78 had CTEPH. Forty-eight patients showed no evidence of CTEPH and included patients with a diagnosis of no $\mathrm{PH}(\mathrm{n}=5)$, and all major forms of $\mathrm{PH}$, including pulmonary arterial hypertension $(\mathrm{n}=18), \mathrm{PH}$ associated with left heart disease $(n=12), P H$ associated with lung disease $(\mathrm{n}=8)$ and $\mathrm{PH}$ multi-factorial $(n=5)$. Six of the MR perfusion image data sets were of non-diagnostic quality. The MR perfusion images correctly identified

Table 1 Summary of diagnostic performance of perfusion scintigraphy, MR perfusion and CTPA in the diagnosis of CTEPH

\begin{tabular}{lll}
\hline & Q scan & MR perfusion* \\
\hline Sensitivity (\%) & $96(95 \% \mathrm{Cl} 0.89 \%$ to $0.99 \%)$ & $97(95 \% \mathrm{Cl} 0.91 \%$ to $0.99 \%)$ \\
Specificity (\%) & $90(95 \% \mathrm{Cl} 0.77 \%$ to $0.97 \%)$ & $92(95 \% \mathrm{Cl} 0.80 \%$ to $0.97 \%)$ \\
Positive predictive value (\%) & $94(95 \% \mathrm{Cl} 0.86 \%$ to $0.98 \%)$ & $95(95 \% \mathrm{Cl} 0.88 \%$ to $0.99 \%)$ \\
Negative predictive value (\%) & $93(95 \% \mathrm{Cl} 0.82 \%$ to $0.99 \%)$ & $96(95 \% \mathrm{Cl} 0.85 \%$ to $0.99 \%)$ \\
Accuracy (\%) & 94 & $95 \quad 9.85 \%$ Cl $0.88 \%$ to $0.99 \%)$ \\
\hline
\end{tabular}

*Inter-observer agreement, $\kappa$ of 0.83 .

CTEPH, chronic thromboembolic pulmonary hypertension; CTPA, CT pulmonary angiography.

Figure 1 (A) Selected coronal images of MR lung perfusion (top) and anterior and posterior view of perfusion scintigraphy images (bottom) in a patient with normal lung perfusion. (B) An example of a patient with chronic thromboembolic pulmonary hypertension with bilateral segmental perfusion defects seen on MR perfusion images (top) and perfusion scintigraphy (bottom).

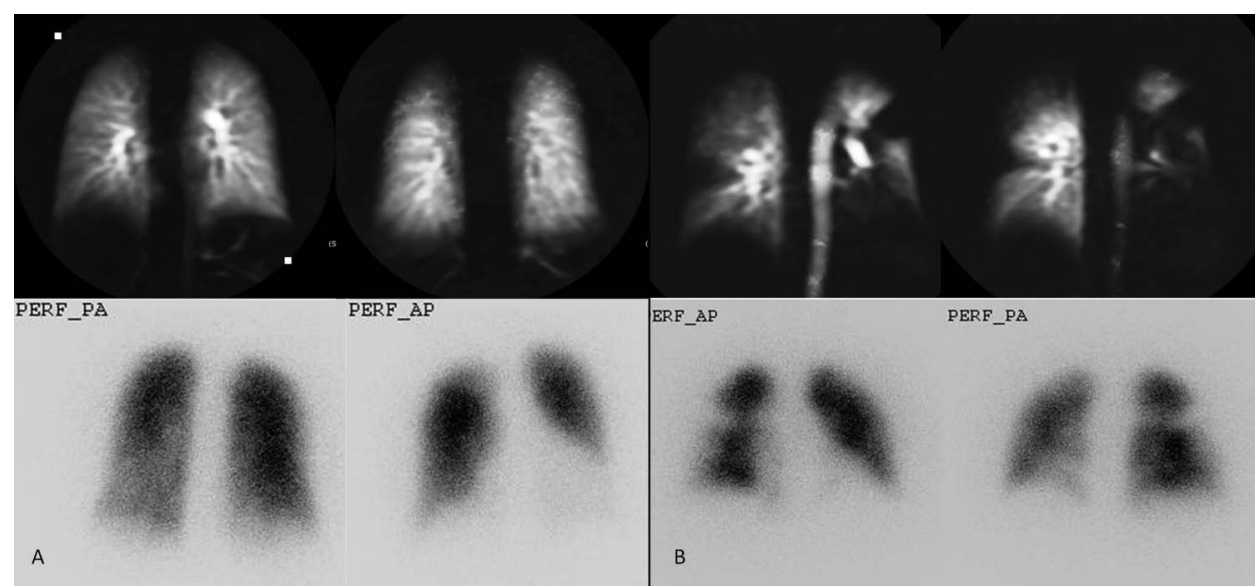


76 patients to have CTEPH giving it a sensitivity of $97 \%$ (95\% CI $0.91 \%$ to $0.99 \%)$ and specificity of $92 \%$ (95\% CI $0.80 \%$ to $0.97 \%$ ) (table 1). The results also showed CTPA, interpreted by the expert pulmonary vascular radiologists, and perfusion scintigraphy to be effective in the diagnosis of CTEPH with a sensitivity of $94 \%(95 \%$ CI $0.85 \%$ to $0.98 \%)$ and $96 \%(95 \%$ CI $0.89 \%$ to $0.99 \%$ ), respectively (figure 1 ). None of the imaging modalities missed any of the patients with surgically accessible disease.

Accurate diagnosis of CTEPH is essential to identify a potentially treatable cause of $\mathrm{PH}$. Here it is demonstrated that 3D lung perfusion MRI, as part of a pulmonary-vascular MRI protocol, has very high sensitivity for the diagnosis of CTEPH similar to that of perfusion scintigraphy and CTPA. The 3D data set allows image reconstruction in any plane, enabling better assessment of regional perfusion abnormalities and has superior temporal and spatial resolution to scintigraphy.

In conclusion, 3D lung perfusion MRI has high sensitivity for CTEPH and complements MR angiography and functional cardiac MRI in a single comprehensive radiation-free imaging modality in the evaluation of patients with suspected CTEPH.

\section{Smitha Rajaram, ${ }^{1}$ Andrew J Swift, ${ }^{1}$}

Adam Telfer, ${ }^{1}$ Judith Hurdman,

Helen Marshall, ${ }^{2,1}$ Eleanor Lorenz, ${ }^{3}$

David Capener, ${ }^{1}$ Christine Davies, ${ }^{4}$

Catherine Hill, ${ }^{4}$ Charlie Elliot, ${ }^{2}$ Robin Condliffe, ${ }^{2}$ Jim M Wild, ${ }^{1}$ David G Kiely ${ }^{2}$

${ }^{1}$ Academic Unit of Radiology, University of Sheffield, Sheffield, UK

${ }^{2}$ Sheffield Pulmonary Vascular Disease Unit, Royal Hallamshire Hospital, Sheffield Teaching Hospitals NHS Foundation Trust, Sheffield, UK

${ }^{3}$ Department of Nuclear Medicine, Sheffield Teaching Hospitals NHS Foundation Trust, Sheffield, UK ${ }^{4}$ Department of Radiology, Sheffield Teaching Hospitals NHS Foundation Trust, Sheffield, UK

Correspondence to Dr David G Kiely, Sheffield Pulmonary Vascular Disease Unit, Royal Hallamshire Hospital, Sheffield Teaching Hospitals NHS Foundation Trust, Glossop Road, Sheffield S10 2JF, UK; david.kiely@sth.nhs.uk

Competing interests None.

Ethics approval Sheffield teaching hospitals.

Provenance and peer review Not commissioned; internally peer reviewed.

- Additional material is published online only. To view please visit the journal online (http://dx.doi.org/10. 1136/thoraxjnl-2012-203020)
To cite Rajaram S, Swift AJ, Telfer A, et al. Thorax 2013;68:677-678.

Received 19 November 2012

Revised 11 December 2012

Accepted 12 December 2012

Published Online First 24 January 2013

Thorax 2013:68:677-678.

doi:10.1136/thoraxjnl-2012-203020

\section{REFERENCES}

1 Swift AJ, Rajaram S, Condliffe R, et al. Diagnostic accuracy of cardiovascular magnetic resonance imaging of right ventricular morphology and function in the assessment of suspected pulmonary hypertension results from the ASPIRE registry. $J$ Cardiovasc Magn Reson 2012;14:40.

2 Amundsen T, Kvaerness J, Jones RA, et al. Pulmonary embolism: detection with MR perfusion imaging of lung-a feasibility study. Radiology 1997;203:181-5.

3 Nikolaou K, Schoenberg SO, Attenberger U, et al. Pulmonary arterial hypertension: diagnosis with fast perfusion MR imaging and high-spatial-resolution MR angiography - preliminary experience. Radiology 2005;236:694-703.

4 Bergin CJ, Hauschildt J, Rios G, et al. Accuracy of MR angiography compared with radionuclide scanning in identifying the cause of pulmonary arterial hypertension. AJR Am J Roentgenol 1997:168:1549-5.

5 Hurdman J, Condliffe R, Elliot CA, et al. Aspire registry: assessing the spectrum of pulmonary hypertension identified at a referral centre. Eur Respir J 2012:39:945-55. 